\title{
MOTIVATEME!: AN ALEXA SKILL TO SUPPORT HIGHER EDUCATION STUDENTS WITH AUTISM
}

\author{
Ami Bakhai, Aurora Constantin and Cristina Adriana Alexandru \\ University of Edinburgh, School of Informatics \\ 10 Crichton Street \\ Edinburgh EH89AB, UK
}

\begin{abstract}
The rate of successful completion of studies for students with Autism Spectrum Disorders (ASD) in Higher Education (HE) is only $40 \%$, which is lower than that for many other students with disabilities. This is caused by a series of challenges encountered by this population in HE. This paper reports on research studies which identified the top challenges faced by HE students with ASD and explored how a Skill (a custom application) for Amazon Alexa Echo Show (AAES) can be designed to support them in overcoming these challenges. A Skill named MotivateMe! was implemented in two iterations for the AAES. During the evaluation study of MotivateMe!, the Skill was perceived by students and experts as being appropriate for students with ASD to deal with distractions and to motivate them to study (which were identified as being the top challenges).
\end{abstract}

\section{KEYWORDS}

Higher Education, Intelligent Virtual Assistants, Autism, User-Centred Design

\section{INTRODUCTION}

In the UK, the number of students with Autism Spectrum Disorders (ASD) at university is increasing (Gurbuz et al. 2019). However less than $40 \%$ of them complete their qualification, which is the lowest rate of success among the other groups of students with disabilities (Gurbuz et al. 2019). Moreover, they often underachieve academically and face a decreased satisfaction of their experience in Higher Education (HE) (Drake 2014). All of these are mainly consequences of a series of difficulties that students with ASD face, such as the change of environment or unstructured teaching (Friedman et al.2013, Howlin \& Moss 2012). Two main categories of challenges have been identified: social and academic (Gurbuz et al. 2019). Social problems include those with relationships and peer-to-peer conversations. On the academic side, problems lie in managing time, creating a routine and being motivated to study (Gurbuz et al. 2019, Jansen et al. 2018, White et al. 2016).

Recently, attention has been increasingly directed toward the benefits of using technology to support autistic people to deal with their difficulties. Some examples of technological solutions developed to help students with ASD in their studies are smartphone applications (Ayres et al. 2013, Bereznak et al. 2012) and wearable technology (Sahin et al. 2018) which targeted understanding social interactions, as well as a Personal Digital Assistant intended to help with multitasking independently (Mechling et al. 2009).

However, literature is scarce on designing Intelligent Virtual Assistants (IVAs), such as Amazon Alexa, to help students in HE overcome their challenges. Available on the market only since five years ago, Amazon Alexa is the most sold and grossing IVA to date (Gartner, 2017). It allows users to interact with it through speech at a level of quality which feels like talking to a human (Gao et al. 2018). A very recent product, Amazon Alexa Echo Show (AAES), is a voice activated assistant with a visual screen which potentially/presumably makes it more useful for ASD students, since most of them are visual learners (Kokina and Kern 2010). The study by (Lopatovska et al. 2019) discusses the interest in AAES for all individuals, including individuals with disabilities such as ASD. (Yu et al. 2018) highlighted the need of looking into the potential of AAES to support students in general, and students with ASD in particular. 
This paper reports on a project which focused on identifying the top challenges faced by HE students with ASD and exploring how a Skill (a custom application for Amazon Alexa) has to be designed to support them overcome these challenges. The following research questions guided our research: RQ1: What are the top challenges that HE students encounter in their studies? RQ2: How can an AAES Skill be best designed to support HE students with ASD in coping with these challenges? RQ3:To what extent does the new technology help HE students with ASD cope with their challenges?

\section{METHODOLOGY OVERVIEW}

The methodology followed four main stages. First, the research literature was reviewed to identify common challenges faced by HE students with ASD, various technology-based applications to support HE students, and guidelines to design technology for the ASD population. The following guidelines were identified to guide the design: G1. predictability (which evokes the feeling of sameness characteristic in autism and reduces anxiety which is a common problem for this population (Baron-Cohen et al. 2007); G2. visuals (due to the fact that most of the autistic individuals are visual learners (Kokina and Kern 2010); G3. customisation (as individuals with autism form a very heterogeneous population and hence tailoring the Skills to meet particular individual needs is essential (Baron-Cohen et al. 2007). Second, the top challenges faced by HE students with ASD were identified and a set of requirements for the AAES Skill were devised (section 3). The third stage, Implementation, involved the technical development of two iterations of the Skill and the first iteration's formative evaluation (section 4). The final stage, Evaluation (section 5), investigated the potential of the new Skill to help ASD students in HE to deal with distractions and be motivated to study (which were identified as being the top challenges).

A User-Centered Design (UCD) framework was adopted, in which users were involved at every stage of the design process (Norman et al. 1986). However, despite HE students with autism being the end users of the new Skill, their vulnerability means that involving them in empirical studies can cause distress (as supported by (Constantin et al. 2019, Boyd-Graber et al. 2006) and also advised by Disability Student Office in the researchers' university), and is moreover very time and resource consuming. Instead, the researchers involved neurotypical (NT) students, following prior study examples with other ASD population groups (e.g. Frauenberger et al. 2012). That ensured that the age-related expertise was brought into the initial design of the technology. Also, HCI experts with experience in usability and ASD were involved to advise what is appropriate to help students with ASD cope with their challenges.

\section{INFORMING THE DESIGN}

This stage included two studies. During the first, semi-structured interviews were conducted with six NT students between the ages of 18 and 24 and four experts in HCI, ASD and Education. The main aims of interviews were to: 1 . understand better the problems faced by students in their daily lives in HE (RQ1), and 2. gather requirements for the Skill (RQ2). The interviews were transcribed and the data was analysed using thematic analysis (Leavy 2014). The most important resulting requirements were mainly related to usability (ease of use, efficiency, predictability, error prevention), and interaction, outlining the need for positive and encouraging interactions which are adapted to the user (G3). The main problems identified by students were the ease of getting distracted and lack of motivation when studying, not being able to start studying or working, and difficulty in remembering content. The second study consisted of a focus group and design workshop with seven NT students aged 21 to 24, with the aims of 1) prioritising the problems faced by students which had been identified in the interviews (RQ1) and 2) gathering more requirements (RQ2). For the latter, two examples of existing Amazon Alexa Skills were shown to the students for inspiration: wikiHow, a knowledge-based Skill which is very popular on AAES, and mathMania, an audio-only very popular educational Skill which proposes mathematical challenges to the users and assesses their answers. The ease of getting distracted and the lack of motivation when studying were identified as the students' top priority problem. The students were asked to design a Skill for this problem, as guided by some questions. The data was analysed using the same methods and in the first study. Most of the requirements from the first study were confirmed, and more requirements were added, the most important of them being the following: 
- On the user interface design: providing visuals which are familiar to the users to show the status of the Skill, as well as textual instructions; both of these matching the audio output; being positive and customised to the user; using a virtual character (VC) to foster emotional attachment to the Skill.

- On the decision elements of the design: the need for creating a routine through fun with a customisable $\mathrm{VC}$, while also monitoring and blocking other websites/apps that may distract the user.

- On use by autistic students: the need for simple, robust and flexible controls fostering independent use without support, with clear and consistent instructions and feedback.

\section{THE MOTIVATEME! SKILL}

The Skill was built using Amazon's proprietary Alexa Skills Kit (ASK) (Amazon 2020). In its first iteration, it allowed users to verbally register to the Skill (if new to it), then set up a new task (e.g. studying for a certain course) with its expected duration, or open an existing task. Each action was supported by on-screen instructions (e.g. Figure 1) and feedback when the action was completed. For each newly created task, the user was able to pair a VC (a tree or a cat, Figure 2) with their task. The more the user worked on their task in proportion to its expected duration, the more the paired $\mathrm{VC}$ would grow on the screen. If the user did not work on the task for a while, the chosen VC would get ill and be unhappy. The user was expected to complete each task in one go. After the first iteration was completed, a formative evaluation with 22 participants (20 NT students and two HCI and Education experts) was carried out. The participants were asked to try the Skill and complete a questionnaire consisting of the System Usability Scale (SUS) (Brooke 1996) as well as three open ended questions. The SUS was intended to assess the Skill's usability, while the open ended questions were meant to elicit suggestions for improving the Skill. A calculation of the SUS score according to (Brooke 1996) and (Sauro 2011) revealed a letter grade of B in usability (mean score of 76). The open ended questions were analysed using open coding (Saldana 2015) and thematic analysis (Leavy 2014) and revealed as main suggestions adding a 'stop' command to stop a task rather than using a timer of the user's expected time, using simpler commands to activate an action and adding the ability to add notes to the task as reminders to e.g. revisit something in the course. The feedback helped to refine the Skill through a second iteration. In this second iteration, the user has been given more independence by being allowed to set up tasks without starting them, and being able to keep working on the same task by stopping and restarting it verbally. The timer was removed, and progress towards the task's total expected duration is calculated based on the difference between the time when the task was stopped and when it was started. While working on the task, the user can ask for statistics which will indicate progress and how much more work is needed to get the VC to grow further. Moreover, as suggested by the participants, notes can now be added to the task and the user can request to see the full list of notes on the screen. A diagram showcasing the actions that are available to the user, in order, in the second iteration of the Skill is available in Figure 3.

\section{EVALUATION}

The final evaluation stage involved a task-based evaluation method followed by a questionnaire with seven NT students (new to this study) and two experts (ASD and HCI, Education). It was conducted online due to the COVID-19 pandemic, which led to the smaller number of participants. The questionnaires included SUS (Brooke 1996) for gathering the participants' views on the task's usability, but also open questions aimed to determine the general user experience with the Skill, the Skill's appropriateness and potential to motivate students with autism (the experts' questionnaire only), and open questions collecting suggestions for the Skill's future development. The data was analysed similarly to the formative evaluation. The results of the SUS indicated a grade of B in usability (mean SUS score of 71.66). Both of the expert participants considered the Skill appropriate and potentially motivational for students with autism: "I would encourage a student with ASD to give it a try [..], this could motivate them to study". They would recommend the Skill both for ASD and NT students. Moreover, all the NT students enjoyed the Skill and predicted that it would be useful for them as well, to help them with their motivation and concentrating on studies. They all mentioned being willing to recommend the Skill to a fellow student: "I would recommend it to some friends who I think would be motivated by caring for a virtual animal/plant”. 
The following were the three most important suggestions:

- Reducing the complexity of verbal interactions (all NT students and experts): A student participant mentioned "Maybe how many words they [the students] are required to say in the interaction and when the device wouldn't understand them, it could be frustrating”.

- Increasing the level of customisation and personalisation to facilitate engagement and encourage motivation (all NT students and experts), through a more varied choice of VCs or a narrative around the VC which is personalised to the student.

- Improving the transparency of the Skill status (3/7 students, $2 / 2$ experts) and available actions ( $5 / 7$ students, $2 / 2$ experts) by including a menu screen or an indication of the different actions possible from the present screen.

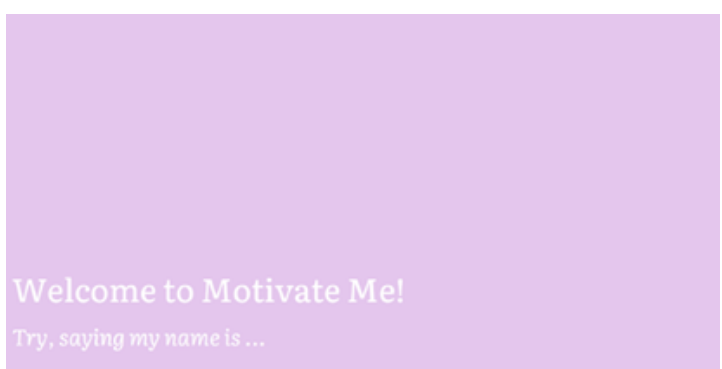

Figure 1. First screen of MotivateMe! and setting up username

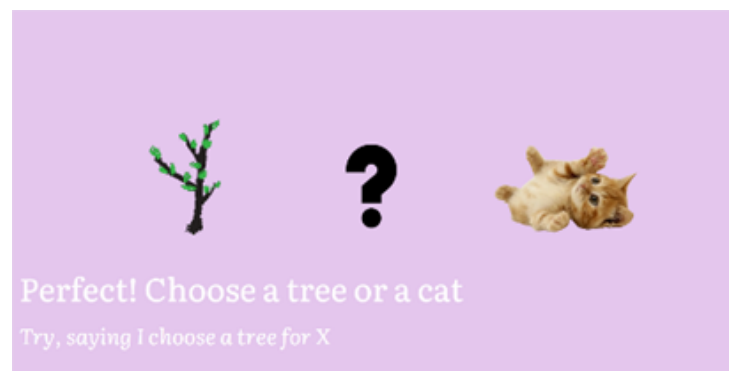

Figure 2. Confirmation of setting up a task and request for pairing it up with a virtual character on MotivateMe!

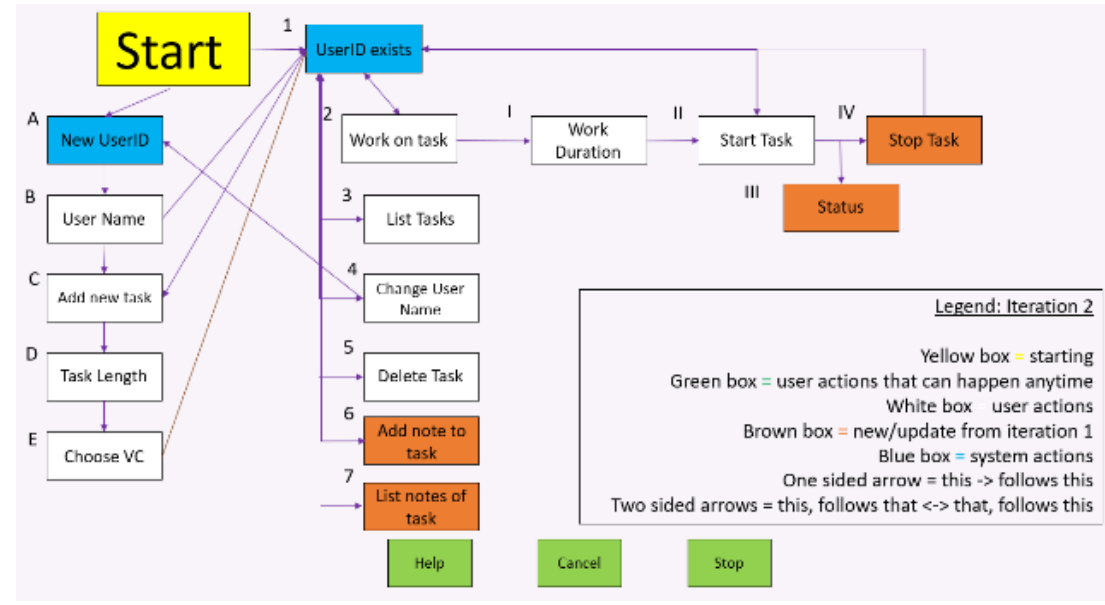

Figure 3. Action Diagram for the second iteration of MotivateMe!

\section{CONCLUSION}

The project focused on researching the best intervention for HE students with ASD through an AAES Skill. The main problems were getting distracted and the lack of motivation to study, and requirements to address them by a Skill were gathered. The AAES Skill MotivateMe! was developed through two iterations, each followed by evaluation with NT students and experts in ASD, HCI and Education. The final evaluation showed a general positive response towards MotivateMe! and demonstrated that the Skill is appropriate for HE students and it also has the potential in supporting HE students with ASD. However, to understand the extent of the potential for students with autism, more research with such students is necessary. The next part of the project includes a larger sample size of participants and participation of autistic students and ASD practitioners. Future work could also involve integration with calendar systems and with course websites such that deadlines 
(e.g. for coursework) are automatically considered, adding reminders to study, and network monitoring such that distracting websites or apps can be automatically blocked (this could not be implemented in the first iterations of the Skill).

\section{REFERENCES}

Ayres, K.M., Mechling, L. and Sansosti, F.J., 2013. The use of mobile technologies to assist with life skills/independence of students with moderate/severe intellectual disability and/or autism spectrum disorders: Considerations for the future of school psychology. Psychology in the Schools, 50(3):259-271.

Baron-Cohen, S., Golan, O., Chapman, E., and Granader, Y. (2007). Transported to a world of emotion. The Psychologist, 20(2):76-77.

Bereznak, S., Ayres, K.M., Mechling, L.C. and Alexander, J.L., 2012. Video self-prompting and mobile technology to increase daily living and vocational independence for students with autism spectrum disorders. Journal of Developmental and Physical Disabilities, 24(3):269-285.

Boyd-Graber, J.L., Nikolova, S.S., Moffatt, K.A., Kin, K.C., Lee, J.Y., Mackey, L.W., Tremaine, M.M. and Klawe, M.M., 2006, April. Participatory design with proxies: developing a desktop-PDA system to support people with aphasia. In Proceedings of the SIGCHI Conference on Human Factors in Computing Systems, 151-160.

Brooke, J., 1996. SUS-A quick and dirty usability scale. Usability evaluation in industry, 189(194):4-7.

Constantin, A., Georgiou, N., Alexandru, C.A. and Korte, J., 2019, September. S2C2: Toward an App to Support Social Story ${ }^{\mathrm{TM}}$ Comprehension Checking in Children with ASD. In IFIP Conference on HCI, 153-160. Springer, Cham.

Drake, S., 2014. College experience of academically successful students with autism. Journal of Autism, 1(1), p.5.

Friedman, N.D., Warfield, M.E. and Parish, S.L., 2013. Transition to adulthood for individuals with autism spectrum disorder: current issues and future perspectives. Neuropsychiatry, 3(2):181.

Gao, Y., Pan, Z., Wang, H. and Chen, G., 2018, October. Alexa, my love: Analyzing reviews of amazon echo. In 2018 IEEE SmartWorld, Ubiquitous Intelligence \& Computing, Advanced \& Trusted Computing, Scalable Computing \& Communications, Cloud \& Big Data Computing, Internet of People and Smart City Innovation (SmartWorld/SCALCOM/UIC/ATC/CBDCom/IOP/SCI),372-380. IEEE.

Gurbuz, E., Hanley, M. and Riby, D.M., 2019. University students with autism: The social and academic experiences of university in the UK. Journal of autism and developmental disorders, 49(2):617-631.

Howlin, P. and Moss, P., 2012. Adults with autism spectrum disorders. The Canadian J. of Psychiatry, 57(5):275-283

Jansen, D., Emmers, E., Petry, K., Mattys, L., Noens, I. and Baeyens, D., 2018. Functioning and participation of young adults with ASD in higher education according to the ICF framework. Journal of Further and HE, 42(2)::259-275.

Kokina, A. and Kern, L., 2010. Social Story ${ }^{\mathrm{TM}}$ interventions for students with autism spectrum disorders: A meta-analysis. Journal of autism and developmental disorders, 40(7):812-826.

Leavy, P. ed., 2014. The Oxford handbook of qualitative research. Oxford University Press, USA.

Lopatovska, I., Rink, K., Knight, I., Raines, K., Cosenza, K., Williams, H., Sorsche, P., Hirsch, D., Li, Q. and Martinez, A., 2019. Talk to me: Exploring user interactions with the Amazon Alexa. Journal of Librarianship and Information Science, 51(4):984-997.

Mechling, L.C., Gast, D.L. and Seid, N.H., 2009. Using a personal digital assistant to increase independent task completion by students with autism spectrum disorder. J. of Autism and developmental disorders, 39(10):1420-1434.

Sahin, N.T., Abdus-Sabur, R., Keshav, N.U., Liu, R., Salisbury, J.P. and Vahabzadeh, A., 2018, September. Case Study of a Digital Augmented Reality Intervention for Autism in School Classrooms: Associated With Improved Social Communication, Cognition, and Motivation via Educator and Parent Assessment. In Frontiers in Education, 3:57.

Saldaña, J., 2015. The coding manual for qualitative researchers. Sage Publications Ltd.

Sauro, J., 2011. Measuring usability with the system usability scale (SUS). [online] Available at https://measuringu.com/sus/ [Accessed $10 \mathrm{Jul}$ 2020]

White, S.W., Elias, R., Salinas, C.E., Capriola, N., Conner, C.M., Asselin, S.B., Miyazaki, Y., Mazefsky, C.A., Howlin, P. and Getzel, E.E., 2016. Students with autism spectrum disorder in college: Results from a preliminary mixed methods needs analysis. Research in Developmental Disabilities, 56, pp.29-40.

Yu, C., Shane, H., Schlosser, R.W., O’Brien, A., Allen, A., Abramson, J. and Flynn, S., 2018. An exploratory study of speech-language pathologists using the Echo Show ${ }^{\mathrm{TM}}$ to deliver visual supports. Advances in Neurodevelopmental Disorders, 2(3):286-292. 